\title{
Concordância entre o índice de adiposidade corporal e percentual de gordura de adultos sedentários de Londrina - PR
}

\author{
Agreement between body fat index and fat percentage \\ of sedentary adults in Londrina - PR
}

\author{
Ferdinando Oliveira Carvalho ${ }^{1}$ \\ Gabriel Gustavo Bergmann² \\ Crisieli Maria Tomeleri ${ }^{3}$ \\ Layane Costa Saraiva ${ }^{4}$ \\ Marcos Vinicius Oliveira Carneiro ${ }^{1}$ \\ Anastácio Neco de Souza Filho ${ }^{1}$ \\ Thaynã Alves Bezerra ${ }^{1}$ \\ Orlando Laitano Lionello Neto ${ }^{1}$
}

\section{RESUMO}

O estudo objetivou verificar a concordância do percentual de gordura com o índice de adiposidade corporal (IAC) e demais indicadores antropométricos de obesidade, e estabelecer pontos de corte para obesidade baseado no IAC de um grupo de brasileiros estratificados por sexo e faixa etária. Participaram do estudo 1.147 adultos sedentários, sendo 451 homens $(27,6 \pm 10,4$ anos $)$ e 696 mulheres $(27,6 \pm 10,4$ anos). Foram analisados o percentual de gordura corporal (\%G), massa corporal total, índice de massa corporal (IMC), circunferência da cintura (CC) e do quadril (CQ), e a relação cintura-quadril (RCQ). Os dados foram processados no SPSS versão 13.0 e Stata ${ }^{\text {tm }}$ versão 9.1. Para ambos os sexos e em todas as faixas etárias foi observado maior correlação do \%G com o IMC e CC e correlação significativa do \%G com todos os índices, sendo maior e positiva para o sexo feminino versus (vs) o masculino: IMC $(0,64$ vs 0,58$)$; RCQ $(0,38$ vs 0,34$)$; CC $(0,65$ vs 0,54$)$; IAC $(0,46$ vs 0,40$)$. Conclui-se que existem correlações significativas entre o $\% \mathrm{G}$ aferido por dobras cutâneas e indicadores antropométricos CC, IMC, IAC e RCQ, e foram estabelecidos pontos de corte para a adiposidade por meio do novo IAC e dos outros indicadores antropométricos para ambos os sexos.

\section{PALAVRAS-CHAVE}

Obesidade; Dobras Cutâneas; Antropometria; Estilo de Vida Sedentário.

\footnotetext{
${ }^{1}$ Universidade Federal do Vale do São Francisco - UNIVASF.

${ }^{2}$ Universidade Federal do Pampa - UNIPAMPA.

${ }^{3}$ Universidade Estadual de Londrina.

${ }^{4}$ Instituto Federal de Educação, Ciência e Tecnologia do Piauí - IFPI.
} 


\section{ABSTRACT}

The study aimed to verify the agreement of the fat percentage with the body adiposity index (IAC) and other anthropometric indicators of obesity, and to establish cutoff points for obesity based on the IAC of a group of Brazilians stratified by sex and age group. 1,147 sedentary adults participated in the study, 451 men (27.6 \pm 10.4 years) and 696 women ( $27.6 \pm 10.4$ years). The percentage of body fat $(\% \mathrm{~F})$, total body mass, body mass index (BMI), waist circumference (WC) and hip (HC), and the waist-hip ratio (WHR) were analyzed. The data were processed using SPSS version 13.0 and Stata ${ }^{\mathrm{tm}}$ version 9.1. For both sexes and in all age groups, there was a greater correlation between $\%$ G with BMI and WC and a significant correlation between $\% \mathrm{G}$ and all indexes, with a higher and positive correlation between females versus (vs) males: BMI (0.64 vs 0.58); WHR (0.38 vs 0.34); WC (0.65 vs 0.54); IAC (0.46 vs 0.40$)$. It is concluded that there are significant correlations between the $\% \mathrm{G}$ measured by skinfolds and anthropometric indicators WC, BMI, IAC and WHR, and cutoff points for adiposity were established using the new IAC and other anthropometric indicators for both sexes.

\section{KEYWORDS}

Obesity; Skin Folds; Anthropometry; Sedentary lifestyle. 


\section{INTRODUÇÃo}

A obesidade é considerada uma epidemia mundial e um dos principais fatores de risco para várias doenças não transmissíveis, atingindo 650 milhões de pessoas de todas as idades e de todos os grupos sociais em todo o mundo (BRASIL, 2020). No Brasil, os dados são preocupantes, especialmente entre os adultos de 25 a 34 anos, nos quais a prevalência de obesos é de 19,8\% (VIGITEL, 2018).

O diagnóstico de excesso de peso e obesidade pode ser realizado de diversas maneiras, seja por meio da utilização de equipamentos de tecnologia avançada (Tomografia Computadorizada, Impedância Bioelétrica-BIA e Absortometria Radiológica de Dupla Energia-DXA) ou por métodos de baixa complexidade e fácil aplicação (Circunferência da Cintura-CC, Espessura de Dobras Cutâneas, Índice de Massa Corporal-IMC, dentre outras). Contudo, o IMC apesar de ser bastante empregado para a classificação do estado nutricional e possíveis riscos à saúde, apresenta divergências e limitações, pois nem sempre quantifica a gordura corporal (MALTA et al., 2012; PRENTICE; JEBB, 2001). E dentre as formas de mensuração do estado de saúde da população adulta, Bergman et al. (2011) formulou o índice de adiposidade corporal (IAC), o qual é calculado tendo como referência a estatura e a circunferência do quadril.

Durante o processo de validação da nova medida, os autores utilizaram dados de percentual de gordura de indivíduos americanos com ascendência hispânica e africana respectivamente, e criaram uma equação que concordou com o percentual de gordura medido por DXA, sem a necessidade da realização de ajustes estatísticos. Contudo, embora o IAC tenha sido fortemente correlacionado com adiposidade da referida população (CAMHI et al., 2011), estudos posteriores apresentaram resultados inconsistentes e contraditórios quanto a sua utilização (FREEDMAN et al., 2012; JOHNSON et al., 2012; SUNG; OH; LEE, 2014; ZHANG et al., 2014), o que reforça a necessidade de novas pesquisas. Além disso, dados já publicados não se referem a estudos com a população brasileira miscigenada, evidenciando-se a necessidade de verificar a relação deste novo índice (IAC) com os demais indicadores de adiposidade corporal, além de averiguar os pontos de corte específicos para o IAC nesta população.

Diante do exposto, o objetivo do estudo foi Verificar a concordância do percentual de gordura com o IAC e demais indicadores antropométricos, e estabe- lecer pontos de corte para obesidade baseado no IAC de um grupo de brasileiros sedentários estratificados por sexo e faixa etária.

\section{MÉTODOS}

Amostra

Participaram do estudo 1.147 indivíduos adultos de Londrina-PR $(60,7 \%$ mulheres $)$ com média de idade $27,6 \pm 10,4$ anos. Para participar do presente estudo os indivíduos precisavam ser sedentários e aparentemente saudáveis. A fim de cumprir com tais requisitos, o tempo em comportamento sedentário foi verificado por meio do Questionário de Nível de Atividade física (IPAQ) versão curta (MATSUDO et al., 2001) e a estratificação de risco pelo American College of Sports Medicine in 1995 (entrevista com análise de sinais e sintomas e/ou fatores de risco cardiopulmonar).

0 tamanho amostral foi calculado com o auxílio do software GPower 3.1, ao considerar um Effect Size = 0,3; $\alpha=0,001$ e; Power $=0,95$, o tamanho requerido de amostra para correlação entre duas variáveis foi de 239 indivíduos. Considerando que no presente estudo foram realizadas quatro correlações simples entre variáveis (\%G vs. IMC, CC, IAC e RCQ), o número necessário para a amostra total foi assumido em 956 indivíduos.

Previamente ao estudo, todos os sujeitos assinaram um termo de consentimento livre e esclarecido, no qual foram informados todos os procedimentos do estudo, bem como possíveis riscos e benefícios do experimento. 0 projeto foi aprovado pelo Comitê de Ética em Pesquisa da universidade local sob o protocolo n. 5931.0.000.441-10.

\section{Avaliação de Indicadores Antropométricos}

A massa corporal total (MCT) foi mensurada em uma balança de plataforma digital (PS 180a, Urano, Brasil) com resolução de 0,1 kg, e a estatura em um estadiômetro de madeira com resolução de $0,1 \mathrm{~cm}$, de acordo com os procedimentos previamente descritos por Gordon et al. (1988). O cálculo do IMC foi realizado a partir da divisão da MCT (em quilos) pela estatura (em metros) elevada à segunda potência. A circunferência da cintura (CC) e do quadril (CQ) foram mensuradas por meio de uma fita métrica metálica inextensível (escala de 0,1 cm, Cescorf, Brasil) conforme as técnicas descritas por Callaway et al. (1988). A relação cintura quadril 
(RCQ) foi calculada por meio da divisão entre a CC e a CQ. Todas as medidas foram realizadas por um único avaliador com experiência nos procedimentos.

\section{Avaliação do Percentual de Gordura (\%G)}

O percentual de gordura corporal (\%G) foi determinado pela técnica de espessura de dobras cutâneas abdominal, peitoral e coxa medial nos homens, e coxa medial, triciptal e supra-ilíaca nas mulheres, de acordo com o protocolo de Slaughter et al. (1984). Após a aferição das dobras, foi calculada a densidade corporal por meio das equações propostas por Jackson \& Pollock (1978) para homens e Jackson, Pollock \& Ward (1980) para mulheres, e em seguida, convertida em \%G pela equação de Siri et al. (1961). O \%G foi classificado para cada indivíduo em acordo com a literatura (LOHMAN, 1992).

Além das medidas de $\%$ G por meio da técnica de dobras cutâneas, esse parâmetro também foi estimado pelo IAC, em acordo com Bergman et al. (2011) com a seguinte fórmula: [circunferência do quadril (cm) / altura $\mathrm{x} \sqrt{ }$ altura $(\mathrm{m})]$ - 18. Este índice foi previamente validado para estimar o \%G através de medidas de DXA (CAMHI et al., 2011) em amostra de americanos encontrando boa concordância e um $\mathrm{R}=0,85(\mathrm{p}<0,001)$ entre ambas as medidas.

\section{Procedimentos Estatísticos}

O teste Kolmogorov-Smirnov foi empregado para análise da distribuição dos dados, que foi considerada simétrica. Estatística descritiva com procedimentos de média e desvio padrão foi utilizada para caracterização da amostra. 0 teste " $\mathrm{t}$ " de student para amostras inde- pendentes foi adotado para comparar diferentes variáveis entre os sexos. A correlação linear de Pearson fora empregada para verificar o grau de associação entre \%G e indicadores antropométricos. Para identificação dos indicadores antropométricos preditores significativos do \%G foi adotada a análise a partir da técnica das curvas Receiver Operating Characteristic - ROC (ERDREICH; LEE, 1981) em que a área total sob a curva ROC foi identificada previamente para os diferentes indicadores antropométricos e com referência ao \%G tradicionalmente mensurado. Para análise deste procedimento foi adotado o intervalo de confiança (IC) de 95\%, no qual quanto maior a área sob a curva ROC, maior o poder discriminatório do respectivo indicador na predição do $\% \mathrm{G}$, sendo o IC determinante da capacidade preditiva dos indicadores selecionados. Para o indicador antropométrico ser considerado como preditor do $\% \mathrm{G}$, o limite inferior do IC $(L i-I C)$ não deve ser $<0,50$ (SCHISTERMAN et al., 2001). A sensibilidade e a especificidade dos indicadores antropométricos foram calculadas através da curva ROC. 0 ponto de corte foi considerado através do valor que melhor apresentou o equilíbrio percentual entre a sensibilidade e a especificidade. Foi considerado o nível de significância de p < 0,05 e os dados foram processados nos pacotes estatísticos SPSS versão 13.0 e Stata $^{\mathrm{tm}}$ versão 9.1 .

\section{RESULTADOS}

A tabela 1 apresenta as características gerais da amostra constatando as diferenças significativas entre os sexos em todas as variáveis, exceto idade ( $\mathrm{p} \leq 0,001)$. Os homens apresentaram resultados superiores nas variáveis de MCT, estatura, IMC, CC, CQ e RCQ. Entretanto, as mulheres apresentaram resultados superiores no IAC e \%G.

Tabela 1. Caracterização da amostra (valores de média, desvio padrão, mínimo e máximo) do presente estudo dividida por sexos ( $\mathrm{n}=1147)$.

\begin{tabular}{lllll}
\hline & $\begin{array}{l}\text { MASCULINO } \\
(\mathrm{n}=451)\end{array}$ & Mín-Máx & $\begin{array}{l}\text { FEMININO } \\
(\mathrm{n}=696)\end{array}$ & Mín-Máx \\
\hline Idade (anos) & $27,6 \pm 10,4$ & $18,0-75,0$ & $27,8 \pm 10,8$ & $18,0-74,0$ \\
MC (Kg) & $76,8 \pm 13,6^{*}$ & $41,6-137,2$ & $59,3 \pm 9,8$ & $38,1-115,5$ \\
Estatura (m) & $1,76 \pm 0,07^{*}$ & $1,53-2,02$ & $1,63 \pm 0,06$ & $1,42-1,85$ \\
IMC (Kg/m²) & $24,5 \pm 3,7^{*}$ & $14,1-43,3$ & $22,0 \pm 3,4$ & $14,9-42,4$ \\
IAC $(\%)$ & $23,2 \pm 3,3^{*}$ & $14,4-38,3$ & $27,4 \pm 3,6$ & $16,4-52,9$ \\
CC $(\mathrm{cm})$ & $84,1 \pm 10,4^{*}$ & $60,8-132,9$ & $73,4 \pm 8,3$ & $56,0-114,8$ \\
CQUAD $(\mathrm{cm})$ & $96,8 \pm 7,1^{*}$ & $78,1-131,8$ & $95,3 \pm 6,8$ & $74,0-130,9$ \\
RCQ $(\mathrm{cm})$ & $0,86 \pm 0,08^{*}$ & $0,58-1,34$ & $0,77 \pm 0,06$ & $0,58-1,09$ \\
$\quad \% G$ & $20,0 \pm 5,9^{*}$ & $3,5-42,3$ & $26,0 \pm 6,4$ & $8,7-45,6$ \\
\hline
\end{tabular}

*Diferença significativa entre os sexos ( $<$ < 0,05). MC: Massa corporal; IMC: Índice de massa corporal; IAC: Índice de adiposidade corporal; CC: Circunferência da cintura; CQUAD: Circunferência de quadril; RCQ: Relação cintura/quadril; \%G: Percentual de gordura. 
Na tabela 2 é apresentada a comparação dos indicadores IAC e IMC com o \%G. Foi possível observar que em ambos os sexos a diferença foi menor entre o IAC e o \%G se comparado com a diferença entre o IMC e o \%G. No entanto, o menor dCohen foi observado para a diferença entre o IAC e \%G do sexo feminino.

Tabela 2. Comparação do IAC com o IMC.

\begin{tabular}{lllll}
\hline & Homens $(\mathrm{n}=451)$ & dCohen & Mulheres $(\mathrm{n}=696)$ & dCohen \\
\hline \% Gordura & $20,0 \pm 5,9$ & & $26,0 \pm 6,4$ \\
IMC & $24,5 \pm 3,7^{*}$ & 0.914 & $22,0 \pm 3,4$ * & -0.781 \\
IAC & $23,2 \pm 3,3^{*}$ & 0.669 & $27,4 \pm 3,6$ * & 0.270 \\
\hline
\end{tabular}

Nota: *Diferença significativa para o \% Gordura (p $\leq 0,001)$; IMC: índice de massa corporal; IAC: índice de adiposidade corporal.

A associação do \%G com IAC na plotagem de Bland \& Altman (1986) para os homens e mulheres está apresentada no gráfico 1. Na figura A observa-se que no sexo masculino a diferença entre as médias foi de $-3,2$ (IC
95\% para a diferença média foi entre 7,$2 ;-13,7$ ) e na figura B, para o sexo feminino, a diferença entre as médias foi de -1,4 (IC 95\% para a diferença média foi entre $9,5 ;-12,3)$.

Gráfico 1. Plotagem de Bland e Altman (1986) entre o \% de Gordura e o IAC para homens ( $\mathrm{n}=451)$ e mulheres $(\mathrm{n}=696)$.

a) Homens

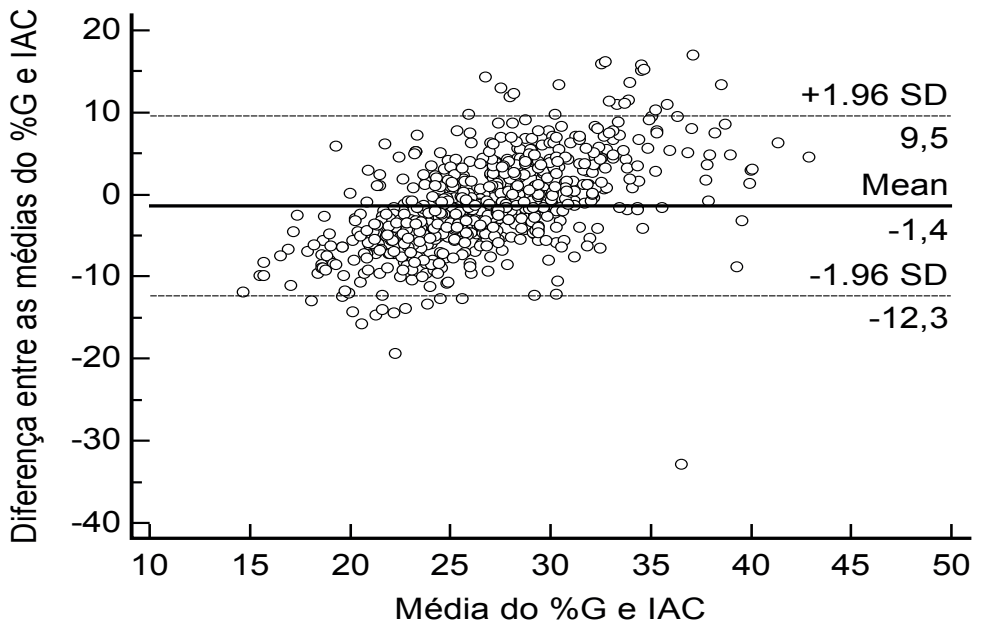

b) Mulheres

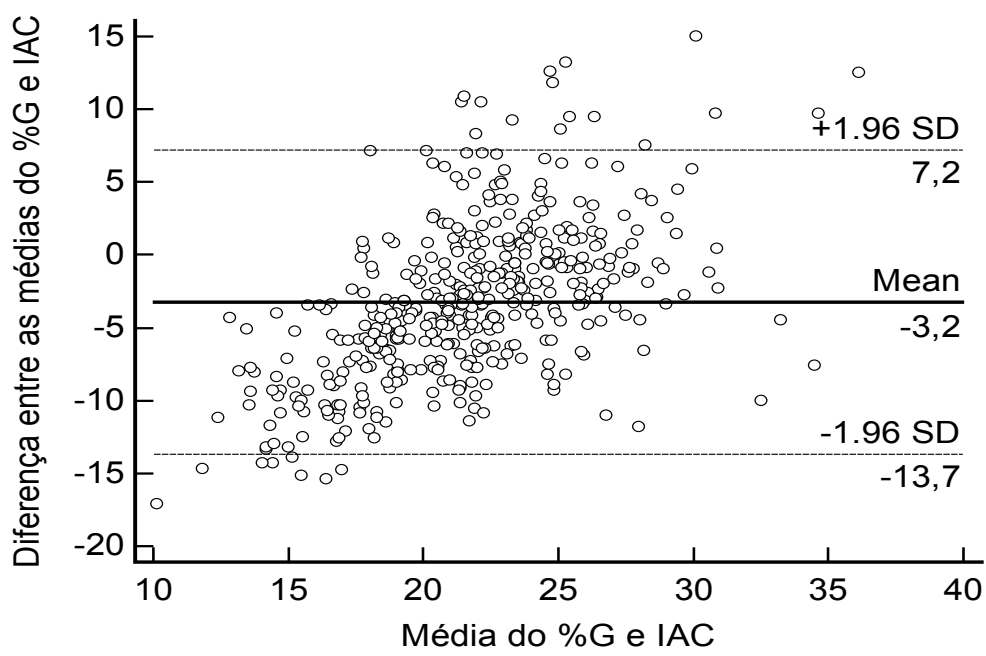


As correlações entre o \%G e os demais indicadores antropométricos estudados (IMC, RCQ, CC e IAC) estão apresentadas na tabela 3. No sexo masculino, pode-se observar que em todas as faixas etárias houve maior correlação do \%G com o IMC e CC. Similarmente, no sexo feminino o \%G também se correlacionou mais efetivamente com IMC e CC em todas as idades. É relevante observar, para ambos os sexos, que a variável RCQ não apresentou correlação significativa com o \%G somente na faixa etária de 41 a 50 anos, e o \%G de gordura se correlacionou de forma significativa com todos os índices, destacando uma correlação maior e positiva para o sexo feminino.

Tabela 3. Resultados por faixa-etária da correlação entre o percentual de gordura (referência) e os demais indicadores antropométricos investigados.

\begin{tabular}{llllll}
\hline \multicolumn{2}{c}{ MASCULINO } & & & & \\
GE (anos) & $\mathrm{N}$ & $\mathrm{IMC}$ & $\mathrm{RCQ}$ & $\mathrm{CC}$ & $\mathrm{IAC}$ \\
\hline $\mathbf{1 8 - 3 0}$ & $\mathbf{2 8 8}$ & $\mathrm{r}=0,59 ; p=0,001$ & $\mathrm{r}=0,35 ; \mathrm{p}=0,001$ & $\mathrm{r}=0,55 ; p=0,001$ & $\mathrm{r}=0,40 ; p=0,001$ \\
$\mathbf{3 1 - 4 0}$ & $\mathbf{7 8}$ & $\mathrm{r}=0,51 ; p=0,001$ & $\mathrm{r}=0,27 ; p=0,01$ & $\mathrm{r}=0,51 ; p=0,001$ & $\mathrm{r}=0,36 ; p=0,001$ \\
$\mathbf{4 1 - 5 0}$ & 30 & $\mathrm{r}=0,54 ; p=0,002$ & $\begin{array}{l}\mathrm{r}=0,2 \quad 9 ; \\
p=0,115^{*}\end{array}$ & $\mathrm{r}=0,54 ; p=0,002$ & $\mathrm{r}=0,38 ; p=0,04$ \\
\hline
\end{tabular}

FEMININO

\begin{tabular}{|c|c|c|c|c|c|c|}
\hline GE (anos) & & $\mathbf{N}$ & IMC & RCQ & CC & IAC \\
\hline $18-30$ & & 388 & $\mathrm{r}=0,58 ; p=0,001$ & $\mathrm{r}=0,36 ; p=0,001$ & $\mathrm{r}=0,62 ; p=0,001$ & $\mathrm{r}=0,42 ; p=0,001$ \\
\hline $31-40$ & $\% G$ & 124 & $\mathrm{r}=0,70 ; p=0,001$ & $\mathrm{r}=0,33 ; p=0,01$ & $\mathrm{r}=0,62 ; p=0,001$ & $\mathrm{r}=0,51 ; p=0,001$ \\
\hline $41-50$ & & 61 & $\mathrm{r}=0,76 ; p=0,002$ & $\begin{array}{l}r=0,44 ; \\
p=0,115^{*}\end{array}$ & $r=0,78 ; p=0,002$ & $\mathrm{r}=0,54 ; p=0,04$ \\
\hline
\end{tabular}

\section{MASCULINO}

\begin{tabular}{|c|c|c|c|c|c|c|}
\hline GE (anos) & & $\mathbf{N}$ & IMC & RCQ & CC & IAC \\
\hline $18-50$ & $\% G$ & 396 & $\mathrm{r}=0,58 ; p=0,001$ & $\mathrm{r}=0,34 ; p=0,001$ & $\mathrm{r}=0,54 ; p=0,001$ & $\mathrm{r}=0,40 ; p=0,001$ \\
\hline
\end{tabular}

\section{FEMININO}

\begin{tabular}{|c|c|c|c|c|c|c|}
\hline GE (anos) & & $\mathbf{N}$ & IMC & RCQ & CC & IAC \\
\hline $18-50$ & $\% G$ & 573 & $r=0,64 ; p=0,001$ & $\mathrm{r}=0,38 ; p=0,001$ & $\mathrm{r}=0,65 ; p=0,001$ & $\mathrm{r}=0,46 ; p=0,001$ \\
\hline
\end{tabular}

*p > 0,05. GE: Grupo etário; \%G: Percentual de gordura; IMC: Índice de massa corporal; RCQ: Relação cintura/quadril; CC: Circunferência da cintura; IAC: Índice de adiposidade corporal.

A tabela 4 referente aos pontos de corte encontrados para os diferentes indicadores antropométricos sob a curva ROC demonstra um significativo poder discriminatório do \%G a partir dos indicadores antropométricos IMC, RCQ CC e IAC para ambos os sexos ( $p<0,001$ ). Contudo, apesar de estatisticamente significativos, o IAC e a RCQ em ambos os sexos mostraram uma discreta fragili- dade para apresentar significativo poder de predição do \%G, com áreas sob a curva ROC e percentual de sensibilidade e especificidade, ligeiramente inferiores aos indicadores antropométricos IMC e CC. Ressalta-se também que em todas as variáveis, pôde-se constatar uma diferença entre os gêneros apresentando valores superiores para o sexo feminino, inclusive para os pontos de corte. 
Tabela 4. Área sob a curva ROC e intervalo de confiança (IC) de 95\%, sensibilidade e especificidade dos pontos de corte (PC) de indicadores antropométricos em referência ao percentual de gordura (\%G) na amostra do sexo masculino $(n=396)$ e feminino $(n=573)$

Referência: Percentual de Gordura*

MASCULINO (> 25\%)

\begin{tabular}{lllllll}
\hline & Área ROC & IC (95\%) & Sensibilidade (\%) & Especificidade (\%) & PC & $\boldsymbol{P}$ \\
\hline IMC & 0,80 & $0,73-0,86$ & 75,0 & 74,4 & 26,0 & 0,001 \\
RCQ & 0,68 & $0,60-0,75$ & 62,5 & 68,5 & 0,89 & 0,001 \\
CC & 0,77 & $0,69-0,83$ & 72,2 & 73,8 & 87,7 & 0,001 \\
IAC & 0,66 & $0,57-0,73$ & 62,5 & 63,9 & 23,8 & 0,001 \\
\hline
\end{tabular}

Referência: Percentual de Gordura*

FEMININO $(>32 \%)$

\begin{tabular}{lllllll}
\hline & Área ROC & IC (95\%) & Sensibilidade (\%) & Especificidade (\%) & PC & $\boldsymbol{P}$ \\
\hline IMC & 0,86 & $0,81-0,90$ & 79,3 & 79,2 & 23,0 & 0,001 \\
RCQ & 0,72 & $0,66-0,77$ & 65,2 & 64,2 & 0,78 & 0,001 \\
CC & 0,87 & $0,83-0,91$ & 79,3 & 80,2 & 77,0 & 0,001 \\
IAC & 0,75 & $0,69-0,81$ & 69,6 & 68,0 & 28,2 & 0,001 \\
\hline
\end{tabular}

*Percentual de gordura elevado segundo Lohman (1992) TG. Advances in body composition assessment. Champaing, Illinois: Human Kinetics Publishers. IMC: Índice de massa corporal; RCQ: Relação cintura/quadril; CC: Circunferência da cintura em centímetros; IAC: Índice de adiposidade corporal (em percentual).

\section{DISCUSSÃO}

Um ponto a ser destacado é que o novo IAC é uma medida equivalente ao \%G obtido a partir da técnica de dobras cutâneas, porém, sem a necessidade de mensurá-las. No presente estudo constatou-se que os valores médios de IAC e \%G foram maiores para as mulheres quando comparado aos homens demonstrando diferenças significativas. Em um estudo realizado por Lichtash et al. (2013), comprovou-se que o IAC apresentou ótima correlação com o \%G em ambos os sexos. Pode-se notar no mesmo estudo que os valores de IAC e \%G foram significativamente maiores em mulheres do que em homens, corroborando com os achados de Zangh et al. (2014) e os da presente pesquisa.

Da mesma forma, na comparação dos indicadores IMC e IAC com o \%G, também se constatou um comportamento diferente entre os sexos, sendo que o IAC subestimou o \%G nas mulheres e opostamente superestimou nos homens, o que já fora relatado em outros estudos (FREEDMAN et al., 2012; JOHNSON et al., 2012; ZHANG et al., 2014) e reforça a necessidade da estratificação dos dados por sexo. Ressalta-se que na presente pesquisa o IAC foi o indicador que mais se aproximou do \%G, quando equiparado com o IMC, principalmente nas mulheres.
Os principais resultados evidenciaram que existem correlações significativas entre o \%G e os indicadores antropométricos IMC, CC e IAC em todas as diferentes faixas etárias estudadas e na amostra total, divididos pelo sexo, sendo possível constatar um significativo poder discriminatório do \%G a partir dos indicadores antropométricos IMC, RCQ CC e IAC para ambos os sexos, embora ainda significativa, com ligeira e menor representatividade para o IAC e o RCQ.

Schulze et al. (2012) correlacionaram o \%G com IAC, IMC, CC e CQ em diabéticos tipo-2 e verificaram que o IMC apresentou maior correlação com o \%G, entretanto, a CC em homens e a CQ em mulheres parecem ser melhores preditores do \%G. Por outro lado, Godoy-Matos et al. (2012) verificaram em mulheres com lipodistrofia parcial, que o IAC foi melhor correlacionado com \%G. Segundo esses autores, o IAC pode ser um indicador mais confiável devido apresentar uma melhor correlação com a leptina que o IMC e por ser sensível em capturar as diferenças de adiposidade em um indivíduo. Sulino et al. (2011) demonstraram uma forte correlação do IMC tanto com o \%G quanto com o IAC, porém, destacam melhor correlação do IAC em sua amostra para ambos os sexos, com exceção somente para o sexo feminino, nas faixas etárias de 41 a 60 anos, onde o IMC apresentou maior 
correlação com dobras cutâneas. Nesse sentido, os mesmos autores descrevem que o IMC pode ter uma maior aplicabilidade em indivíduos com maior \%G.

Acredita-se que parte destas discrepâncias possam ser creditadas às diferentes características da amostra, bem como a distinção dos métodos utilizados para a determinação do \%G corporal. No entanto, frente às constantes críticas postuladas a utilização do IMC (PRENTICE; JEBB, 2001; BERGMAN et al. 2011) para a determinação da gordura, parece que o IAC pode apresentar uma vantagem em relação ao IMC, já que o mesmo foi efetivo na predição da gordura corporal.

Por meio do levantamento bibliográfico, espera-se que esse seja o primeiro estudo a apresentar o padrão de sensibilidade e especificidade de um ponto de corte para predição da obesidade a partir do novo IAC (\%) em amostra de aproximadamente 1000 indivíduos brasileiros de ambos os sexos. Ressaltando que, apesar de todos os indicadores antropométricos terem demonstrado um bom poder discriminatório para predizer \%G, o IAC apresentou valores da área sob a curva ROC e percentual de sensibilidade e especificidade menores do que o IMC, concordando com os dados de Sung et al. (2014). Da mesma forma, López et al. (2012) concluíram que a capacidade discriminatória do IMC, medida pela curva ROC, foi maior que a do IAC, corroborando que o IAC embora apresente a vantagem de não considerar o peso para o \%G, o mesmo geralmente não consegue superar as limitações do IMC (FREEDMAN et al., 2012; ZHANG et al., 2014; SUN et al., 2013).

0 padrão de corte para o IAC determinado pelo presente estudo foi de 23,8 e 28,2 para homens e mulheres respectivamente. Esses achados contribuem com o estudo anteriormente citado, no qual os autores encontraram valores mais elevados para o sexo feminino em relação ao sexo masculino (FREEDMAN et al., 2012).
Esses resultados acarretam importantes informações e aplicação prática para os profissionais da área da saúde que realizam avaliação física antropométrica em seus alunos e/ou pacientes, pois comumente vem aumentado a prevalência de pacientes com diferentes fatores de risco, dentre os quais destaca-se a obesidade e suas complicações (MOREIRA et al., 2008). Nesse sentido, a praticidade em utilizar os pontos de corte dos indicadores propostos neste trabalho (IMC, CC, IAC e RCQ) constitui em procedimentos de fácil aplicabilidade e grande importância clínica para futuras intervenções preventivas e terapêuticas no campo da obesidade. Tais condutas tornam-se ainda mais relevantes na avaliação da população brasileira, a qual tem aumentado a prevalência de obesidade nos últimos 20 anos (LIMA et al., 2015), como demonstrado no estudo de Malta et al. (2014).

Uma das limitações do presente estudo foi não ter utilizado medidas de \%G por meio do padrão ouro, DXA, como destacado na literatura. Entretanto, a validade e a reprodutibilidade do \%G por medidas de dobras cutâneas tem sido confirmada por Neves et al. (2013).

Conclui-se que existem correlações significativas entre o \%G medido por dobras cutâneas e os indicadores antropométricos IAC, CC, IMC, e RCQ em ambos os sexos. E a partir desses resultados, foram estabelecidos pontos de corte para obesidade, especialmente pelo novo índice (IAC), tanto no sexo masculino como no feminino com percentuais de sensibilidade e especificidade equilibrados.

Novos estudos são necessários a fim de testar a validade do IAC numa maior amplitude da população brasileira com diferentes condições (saudáveis vs patológicos) nas diferentes faixas etárias e utilizando medidas de referência com padrão ouro na avaliação. 


\section{REFERÊNCIAS}

BERGMAN, R. N. et al. A better index of body adiposity. Obesity, v. 19, n. 5, p. 1083-1089, 2011.

BRASIL. Ministério da Saúde. 04/3 - Dia Mundial da Obesidade. 2020. Disponível em: <http://bvsms.saude.gov.br/ultimas-noticias/3134-04-3-dia-mundial-da-obesidade>. Acesso em: 01 jul. 2020

CALLAWAY, C. W. et al. Circumferences In: LOHMAN, T. G. et al (Org). Anthropometric standardization reference manual. Champaign, IL: Human Kinetics Books, 1988.

CAMHI, S. M. et al. The relationship of waist circumference and BMI to visceral, subcutaneous, and total body fat: sex and race differences. Obesity, v. 19, n. 2, p. 402-408, 2011.

ERDREICH, L. S.; LEE, E. T. Use of relative operating characteristic analysis in epidemiology: a method for dealing with subjective judgement. American journal of epidemiology, v. 114 n. 5, p. 649-662, 1981.

FREEDMAN, D. S. et al. The body adiposity index (hip circumference $\div$ height 1.5 ) is not a more accurate measure of adiposity than is BMI, waist circumference, or hip circumference. Obesity, v. 20 , n. 12 , p. $2438-2444,2012$

GODOY-MATOS, A. F. et al. A new method for body fat evaluation, body adiposity index, is useful in women with familial partial lipodystrophy. Obesity, v. 20, n. 2, p. 440-443, 2012.

GORDON, C. C.; CHUMLEA, W. C.; ROCHE, A. F. Stature, recumbent length, and weight. In: LOHMAN, T. G. et al (Org). Anthropometric standardization reference manual. Champaign: Human Kinetics Books, 1988. p. 3-8.

JACKSON, A. S.; POLLOCK, M. L. Generalized equations for predicting body density of men. British journal of nutrition, $\mathrm{v}$ 40, n. 3, p. 497-504, 1978

JACKSON, A. S.; POLLOCK, M. L.; WARD, A. N. N. Generalized equations for predicting body density of women. Medicine and science in sports and exercise, v. 12, n. 3, p. 175-181, 1980.

JOHNSON, W. et al. Concordance of the recently published body adiposity index with measured body fat percent in EuropeanAmerican adults. Obesity, v. 20, n. 4, p. 900-903, 2012.

LICHTASH, C. T. et al. Body adiposity index versus body mass index and other anthropometric traits as correlates of cardiometabolic risk factors. PloS one, v. 8, n. 6, 2013.

LIMA, N. P. et al. Evolução do excesso de peso e obesidade até a idade adulta, Pelotas, Rio Grande do Sul, Brasil, 1982-2012. Cadernos de Saúde Pública, v. 31, p. 2017-2025, 2015.

LOHMAN, T. G. Advances in body composition assessment. Current issues in exercise science series. Monograph, n. 3, 1992.

LÓPEZ, A. A. et al. Body adiposity index utilization in a Spanish Mediterranean population: comparison with the body mass index. PloS one, v. 7, n. 4, 2012.
MALTA, D. C. et al. Evolução anual da prevalência de excesso de peso e obesidade em adultos nas capitais dos 26 estados brasileiros e no Distrito Federal entre 2006 e 2012. Revista Brasileira de Epidemiologia, v. 17, p. 267-276, 2014.

MATSUDO, S. et al. Questionário internacional de atividade física (IPAQ): estudo de validade e reprodutibilidade no Brasil. Revista Brasileira de Atividade Física \& Saúde, v. 6, n. 2, p. $5-18,2001$.

MOREIRA, S. R. et al. Predicting insulin resistance in children anthropometric and metabolic indicators. Jornal de pediatria, v. 84, n. 1, p. 47-52, 2008.

NEVES, E. B. et al. Comparação do percentual de gordura obtido por bioimpedância, ultrassom e dobras cutâneas em adultos jovens. Revista Brasileira de Medicina do Esporte, v. 19, n. 5, p. 323-327, 2013.

PRENTICE, A. M.; JEBB, S. A. Beyond body mass index. Obesity reviews, v. 2, n. 3, p. 141-147, 2001.

SCHISTERMAN, E. F. et al. Statistical inference for the area under the receiver operating characteristic curve in the presence of random measurement error. American Journal of Epidemiology, v. 154, n. 2, p. 174-179, 2001.

SCHULZE, M. B. et al. Body adiposity index, body fat content and incidence of type 2 diabetes. Diabetologia, v. 55, n. 6, p. 1660$1667,2012$.

SIRI, W. E. et al. Body composition from fluid spaces and density: analysis of methods. Techniques for measuring body composition, v. 61, p. 223-244, 1961.

SLAUGHTER, M. H. et al. Influence of maturation on relationship of skinfolds to body density: a cross-sectional study. Human biology, v. 56, n. 4, p. 681-689, 1984.

SULINO, R. M. et al. Comparação entre o índice de adiposidade corporal e a avaliação da composição corporal através de medidas de dobras cutâneas. Coleção Pesquisa em Educação Física, v. 10, n. 1, p. 63-8, 2011.

SUN, G. et al. Concordance of BAI and BMI with DXA in the Newfoundland population. Obesity, v. 21, n. 3, p. 499-503, 2013.

SUNG, Y. A.; OH, J. Y.; LEE, H. Comparison of the body adiposity index to body mass index in Korean women. Yonsei medical journal, v. 55, n. 4, p. 1028-1035, 2014.

VIGITEL, Brasil. Saúde suplementar: vigilância de fatores de risco e proteção para doenças crônicas por inquérito telefônico. Agência Nacional de Saúde Suplementar. Brasília: Ministério da Saúde, 2018.

ZHANG, Z. Q. et al. The validity of the body adiposity index in predicting percentage body fat and cardiovascular risk factors among Chinese. Clinical endocrinology, v. 81, n. 3, p. 356-362, 2014. 